\title{
Atuação do enfermeiro na gestação de alto risco: uma revisão sistemática
}

\author{
The role of nurses in high-risk pregnancy: a systematic review \\ El papel de las enfermeras en el embarazo de alto riesgo: una revisión sistemática
}

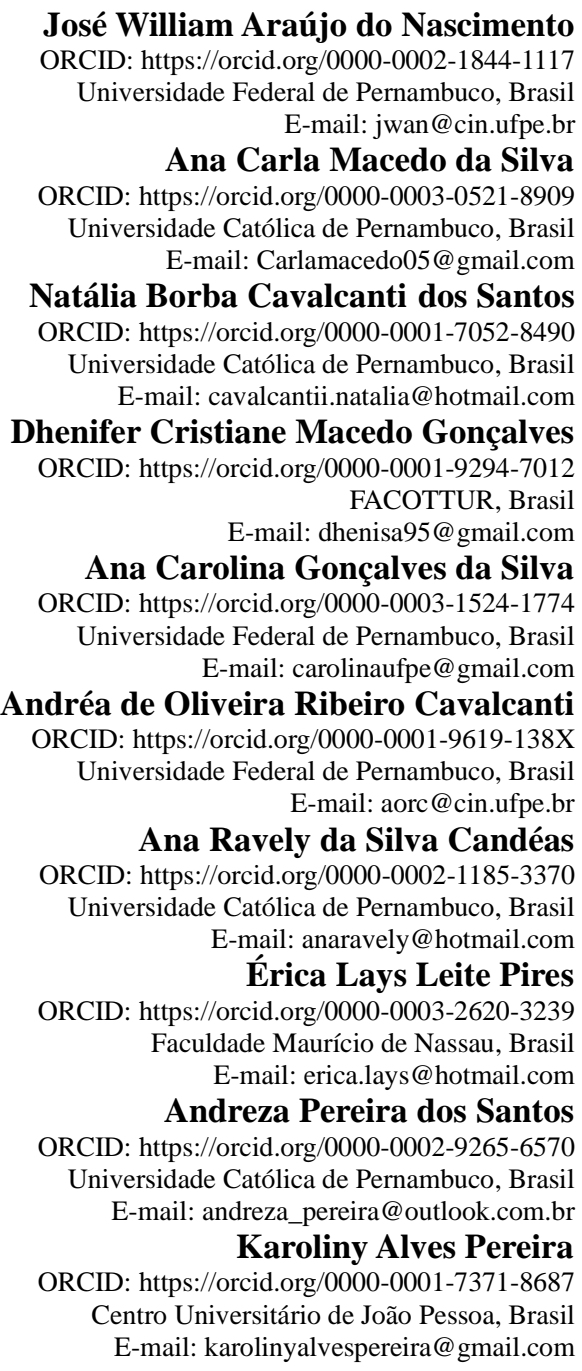

\begin{abstract}
Resumo
Objetivo: Identificar na literatura científica as formas de atuação do enfermeiro à gestantes de alto risco. Metodologia: Revisão sistemática da literatura baseada no método Preferred Reporting Items for Systematic Reviews e MetaAnalysis (PRISMA), realizada nas bases de dados eletrônicas BDENF, Lilacs, Pubmed e Web of Science, por meio dos seguintes descritores: "Gravidez de alto risco", "Cuidados de enfermagem" e "Enfermagem obstétrica". Foram incluídos artigos completos, disponíveis em português, inglês ou espanhol, publicados entre janeiro de 2016 a outubro de 2021. Resultados: 11 artigos compuseram a amostra final deste estudo. Referente a atuação do enfermeiro na gestação de alto risco, a partir da amostra de artigos foi possível elencar os seguintes eixos temáticos: 1. Implementação da Sistematização da Assistência de Enfermagem (SAE) no plano terapêutico das gestantes de risco; 2. Utilizar preceitos preconizados pelo Ministério da Saúde a fim de garantir um atendimento humanizado acolhimento: apoio emocional e informacional; 3. Realizar a avaliação e monitoramento do risco gestacional e executar os cuidados técnicos-científicos no tratamento das doenças associadas ao risco gestacional e durante o parto e puerpério; 4. Realizar a educação em saúde de forma constante nas gestantes de alto risco. Conclusão: Verificou-se que o cuidado à gestante de alto risco ficou evidente por meio de um pré-natal de qualidade, a partir do plano de
\end{abstract}


cuidado individualizado organizado pelo enfermeiro, sob o apoio da família e por meio do uso da Sistematização da Assistência de Enfermagem, de tecnologias leves e pelo processo de educação em saúde.

Palavras-chave: Cuidados de enfermagem; Cuidado pré-natal; Gravidez.

\begin{abstract}
Objective: To identify in the scientific literature the ways in which nurses work with high-risk pregnant women. Methodology: Systematic literature review based on the Preferred Reporting Items for Systematic Reviews and MetaAnalysis (PRISMA) method, performed in the electronic databases BDENF, Lilacs, Pubmed and Web of Science, using the following descriptors: "High-risk pregnancy", "Care of nursing" and "Obstetrical nursing". Full articles were included, available in Portuguese, English or Spanish, published between January 2016 and October 2021. Results: 11 articles comprised the final sample of this study. Regarding the role of nurses in high-risk pregnancy, from the sample of articles, it was possible to list the following thematic axes: 1. Implementation of the Nursing Care Systematization (SAE) in the therapeutic plan for pregnant women at risk; 2. Use precepts recommended by the Ministry of Health in order to guarantee humanized care - reception: emotional and informational support; 3 . Carry out the assessment and monitoring of gestational risk and carry out technical-scientific care in the treatment of diseases associated with gestational risk and during childbirth and puerperium; 4 . Conduct health education constantly in high-risk pregnant women. Conclusion: It was found that care for high-risk pregnant women was evident through quality prenatal care, based on the individualized care plan organized by the nurse, under the support of the family and through the use of Systematization of Care of Nursing, of light technologies and by the process of health education.
\end{abstract}

Keywords: Nursing care; Prenatal care; Pregnancy.

\title{
Resumen
}

Objetivo: Identificar en la literatura científica las formas en que las enfermeras trabajan con mujeres embarazadas de alto riesgo. Metodología: Revisión sistemática de la literatura basada en el método Preferred Reporting Items for Systematic Reviews and Meta-Analysis (PRISMA), realizado en las bases de datos electrónicas BDENF, Lilacs, Pubmed y Web of Science, utilizando los siguientes descriptores: "Embarazo de alto riesgo", "Atención de enfermería" y "Enfermería obstétrica". Se incluyeron artículos completos, disponibles en portugués, inglés o español, publicados entre enero de 2016 y octubre de 2021. Resultados: 11 artículos conformaron la muestra final de este estudio. En cuanto al papel de las enfermeras en el embarazo de alto riesgo, de la muestra de artículos fue posible enumerar los siguientes ejes temáticos: 1. Implementación de la Sistematización de la Atención de Enfermería (SAE) en el plan terapéutico de gestantes en riesgo; 2. Utilizar los preceptos recomendados por el Ministerio de Salud para garantizar una atención humanizada - recepción: apoyo emocional e informativo; 3. Realizar la evaluación y seguimiento del riesgo gestacional y realizar la atención técnico-científica en el tratamiento de enfermedades asociadas al riesgo gestacional y durante el parto y puerperio; 4. Realizar educación sanitaria de forma constante en mujeres embarazadas de alto riesgo. Conclusión: Se encontró que la atención a la gestante de alto riesgo se evidenció a través de una atención prenatal de calidad, basada en el plan de atención individualizado organizado por la enfermera, bajo el apoyo de la familia y mediante el uso de la Sistematización de la Atención de Enfermería, de luz tecnologías y por el proceso de educación para la salud.

Palabras clave: Atención de enfermería; Atención prenatal; Embarazo.

\section{Introdução}

A gravidez é um fenômeno fisiológico que ocorre sem complicações na maioria dos casos, mas que pode envolver alguns riscos em algumas gestantes. A gravidez é definida como de alto risco quando a probabilidade de um resultado adverso para a mulher ou para o feto é maior do que o esperado para a população em geral, e há fatores de risco ou determinantes (Ricci, 2015). A maioria dos riscos está relacionada a condições pré-existentes ou complicações da gravidez por causas orgânicas, biológicas, químicas e ocupacionais, mas também a condições sociais e demográficas desfavoráveis (Wildschut, 2011; Brasil, 2013).

No Brasil, 20\% das gestações são classificadas como de alto risco, caracterizadas por algum distúrbio de saúde que ameace a vida da mãe e / ou do feto, proveniente do processo de gestação ou outro problema anterior agravado durante a gravidez (Brasil, 2012; Ricci, 2015). Esse grupo de mães requer suporte especializado, que abrangerá todos os níveis de complexidade e fornecerá o diagnóstico e os procedimentos terapêuticos necessários (Brasil, 2012).

Grande parte das gestações de alto risco estão relacionadas a alguns transtornos, sendo mais frequentes as síndromes hipertensivas da gravidez e o diabetes mellitus gestacional, onde aproximadamente 3/4 das mortes maternas no mundo ocorrem 
por causas obstétricas diretas (Salge et al., 2017). Quanto às patologias que podem acometer a mulher na gestação, algumas aparecem com maior frequência no primeiro e segundo trimestre, sendo elas as hemorragias, hiperêmese gravídica, abortamento e gravidez ectópica. Outras já aparecem no terceiro trimestre, como placenta prévia, descolamento prematuro da placenta, trabalho de parto prematuro e pré-eclâmpsia/eclampsia (Rios \& Vieira, 2007).

Neste sentido, o cuidado a estas gestantes é de suma importância; a atenção à saúde da mulher durante o ciclo gravídico-puerperal é definida pelo Programa Brasileiro de Humanização no Pré-Natal e Nascimento (PHPN) e pela Rede Cegonha (Brasil, 2002; Giovanni, 2013). No que se refere à gravidez de alto risco, essas orientações ditam a avaliação dos riscos clínicos e obstétricos em todas as consultas, bem como a garantia de assistência médica e acesso ambulatorial e hospitalar em unidade de referência (Errico et al., 2018). O manual técnico do Ministério da Saúde para a gestação de alto risco orienta a equipe assistencial no diagnóstico e tratamento das complicações e padroniza os procedimentos, contribuindo assim para um suporte mais estreito e eficaz (Brasil, 2012).

A gestante de alto risco deve ter seu acompanhamento de pré-natal realizado pela atenção secundária e serviços especializados, e simultaneamente pela Atenção Primária à Saúde (APS) de forma integrada (Ferreira Junior et al., 2017; Soncini et al., 2019). No pré-natal de alto risco, o Ministério da Saúde preconiza o atendimento materno por equipe multiprofissional, que inclui enfermeiros; este é um profissional fundamental nessa área de atuação, sendo responsável pela assistência de enfermagem em seus diversos níveis de apoio assistencial, com competência técnica para desempenhar funções preventivas, promocionais, protetoras e reabilitadoras (Alves et al., 2021).

As perspectivas para o enfermeiro na área de obstetrícia são possíveis devido à ambientação do ambiente de trabalho do profissional, amparada pela resolução do Conselho Federal de Enfermagem (COFEN) nº 0477, de 14 de abril de 2015, que explica a atuação do enfermeiro no cuidado à gestante sendo a mãe, a parturiente e a puérpera uma das funções que, entre outras, incluem as consultas de enfermagem obstétrica e direcionam a assistência de enfermagem às pacientes obstétricas graves (Brasil, 2015).

Diante deste contexto, conforme Amorim et al. (2017), não existem dúvidas que, a assistência à gestante de alto risco necessita de capacitação, habilidade e aptidão dos profissionais no manejo de situações de alerta, emergenciais ou potencialmente complicadas durante o ciclo gravídico puerperal, sendo imprescindível que o profissional enfermeiro esteja assistindo em todos os níveis do sistema de saúde, a fim de corroborar com a precisão diagnóstica, com orientações e acolhimento psicoemocional, trabalhando em prol da prevenção e promoção da saúde da gestante de alto risco e do feto.

Nesta perspectiva, existem poucos estudos sobre a atuação do enfermeiro nas gestações de alto risco; isso porque as gestações de alto risco representam a taxa mais baixa entre as gestantes e por causa da reconhecida prevalência do trabalho do enfermeiro no pré-natal e nas gestações de baixo risco. Percebe-se que a produção de conhecimento e compreensão nesta área é relevante, visto que as gestações de alto risco são eventos complexos que carecem de atenção especializada e embasada em evidências científicas. Somando-se a isso, melhorar a qualidade da atenção à saúde da dupla mãe-filho é um dos Objetivos de Desenvolvimento do Milênio das Nações Unidas; ao mesmo tempo, o ciclo gravídico-puerperal é um dos eixos da Agenda Nacional de Prioridades de Pesquisa em Saúde. Desta forma, este estudo objetivou identificar na literatura científica as formas de atuação do enfermeiro à gestantes de alto risco.

\section{Metodologia}

Este estudo foi conduzido sob a metodologia de uma revisão sistemática da literatura com escopo descritivo, realizada com base no método Preferred Reporting Items for Systematic Reviews e Meta-Analysis (PRISMA) (Moher et al., 2015).

Foi seguida as seguintes etapas, idealizada por Pati e Lorusso (2018): 1) Definição da questão norteadora e objetivos 
da pesquisa; 2) Estabelecimento dos critérios de inclusão e exclusão/ amostragem dos estudos; 3) Busca na literatura; 4) Categorização e análise dos estudos; 5) Apresentação e discussão dos resultados da amostra e; 6) Apresentação e síntese do conhecimento. A questão norteadora deste estudo incide em: "Quais são as principais atividades desenvolvidas pelo enfermeiro na gestação de alto risco?" A investigação dos estudos ocorreu nas seguintes bases de dados: Base de Dados de Enfermagem (BDENF), Literatura Latino-americana e do Caribe em Ciências da Saúde (LILACS), Nacional Institute of Medicine (NIH-PUBMED) e Web of Science, no período de setembro a novembro de 2021. As bases de dados foram selecionadas pela sua grande abrangência de estudos.

Realizou-se consulta ao Medical Subject Headings (MeSH) e ao Descritores em Ciências da Saúde (DeCS). Foram utilizados os seguintes descritores: "Gravidez de Alto Risco", "Cuidados de Enfermagem", "Enfermagem Obstétrica", "Pregnancy, High-Risk", "Nursing Care" e "Obstetric Nursing".

Os artigos selecionados atenderam aos seguintes critérios de inclusão: textos completos, disponíveis em português, inglês ou espanhol, publicados entre janeiro de 2016 a outubro de 2021. Foram excluídos artigos duplicados nas bases de dados (duplicatas), estudos de revisão e pesquisas com temática não relevante a questão norteadora da pesquisa.

É importante mencionar que não foram incluídos na amostra artigos que apenas descrevessem o perfil, fatores de risco, aspectos epidemiológicos ou sentimentos e vivências das gestantes de alto risco e que não comentassem sobre os cuidados de enfermagem do público, uma vez que esses estudos não atenderam aos requisitos desta pesquisa.

Para garantir o registro do conjunto de informações relevantes ao tema utilizou-se um formulário elaborado para o presente estudo, que adotou as orientações fornecidas pela Cochrane Collaboration (Higgins \& Green, 2011) no que se refere ao conteúdo e à estrutura, com as seguintes variáveis: dados de identificação geral (título, autores, periódico e sua classificação na Coordenação de Aperfeiçoamento de Pessoal de Nível Superior - CAPES, ano de publicação, delineamento metodológico (tipo/abordagem do estudo e nível de evidência), objetivos e principais resultados.

Após a aplicação dos filtros de pesquisa nas bases de dados, inicialmente foram encontrados 798 artigos. Os artigos duplicados (241) foram registrados apenas uma vez, totalizando em 557 para leitura dos títulos e resumos. Nesta etapa foram excluídas 447 publicações que não tinham abordagem relevante a temática deste estudo e 39 estudos de revisão. Sendo assim, 71 publicações foram selecionadas para leitura na íntegra, porém 60 foram excluídas por não apresentarem atuações específicas dos enfermeiros a gestantes de alto risco. Desta forma, 11 artigos constituíram a amostra final, conforme explicitado na Figura 1. 
Figura 1: Fluxograma do processo de seleção do estudo, Recife (PE), Brasil, 2021.

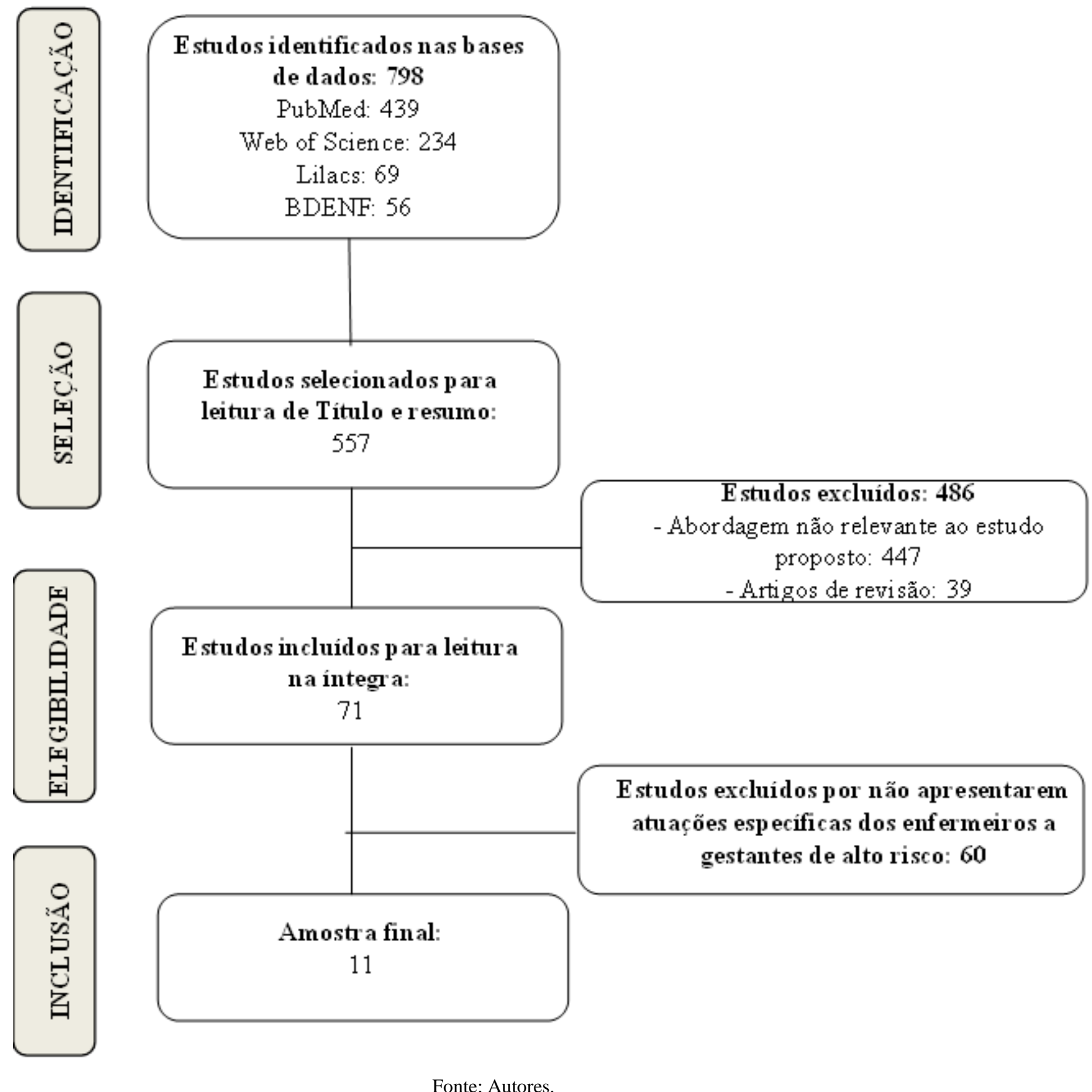

Os artigos selecionados foram submetidos à classificação do nível de evidência, a partir do instrumento de Classificação Hierárquica das Evidências para Avaliação dos Estudos (Stillwell et al., 2010). Segundo esta classificação, os níveis I e II são considerados evidências fortes, III e IV moderadas e V a VII fracas. Uma vez que a maioria dos estudos analisados apresentou diferenças metodológicas significativas, a realização da metanálise foi inviabilizada. Portanto, na presente revisão sistemática realizamos uma síntese qualitativa para apresentação dos dados.

Este estudo preservou os aspectos éticos de tal forma que todos os autores das publicações analisadas foram referenciados apropriadamente, mediante a Lei de Direitos Autorais no 9.610 de 19 de Fevereiro de 1998 (Brasil, 1998). 


\section{Resultados}

A amostra final foi composta por 11 artigos, de tal modo que estes estudos foram publicados com maior frequência em 2020 (n: 03). Em relação à abordagem dos artigos encontrados, verifica-se que a maior parte utilizou o estudo transversal qualitativo (n: 08), enquanto os demais foram o transversal quantitativo (n: 01), retrospectivo (n: 01) e ensaio clínico randomizado (n: 01). Desta forma verifica-se que o nível de evidência mais prevalente nos estudos foi o fraco (VI), embora apenas um estudo tenha apresentado um nível de evidência forte (II).

Referente a atuação do enfermeiro na gestação de alto risco, a partir da amostra de artigos foi possível elencar os seguintes eixos temáticos: 1. Implementação da Sistematização da Assistência de Enfermagem (SAE) no plano terapêutico das gestantes de risco; 2. Utilizar preceitos preconizados pelo Ministério da Saúde a fim de garantir um atendimento humanizado acolhimento: apoio emocional e informacional; 3. Realizar a avaliação e monitoramento do risco gestacional e executar os cuidados técnicos-científicos no tratamento das doenças associadas ao risco gestacional e durante o parto e puerpério, utilizando tecnologias leves; 4. Realizar a educação em saúde de forma constante nas gestantes de alto risco.

A fim de apresentar os resultados desta revisão em um formato sinóptico, elaborou-se um quadro síntese (Quadro 1) que enfatiza informações relevantes dos estudos selecionados.

Quadro 1: Síntese dos estudos avaliados, Recife (PE), Brasil, 2021.

\begin{tabular}{|c|c|c|c|c|c|}
\hline ID & $\begin{array}{l}\text { Autores } \\
\text { (ano) }\end{array}$ & $\begin{array}{l}\text { Periódico } \\
\text { (Qualis) }\end{array}$ & $\begin{array}{c}\text { Tipo de estudo } \\
\left(\mathrm{NE}^{*}\right)\end{array}$ & Objetivos & Principais resultados \\
\hline 01 & $\begin{array}{l}\text { Araújo et } \\
\text { al. (2016) }\end{array}$ & $\begin{array}{l}\text { Revista Latino- } \\
\text { Americana de } \\
\text { Enfermagem (A1) }\end{array}$ & $\begin{array}{c}\text { Ensaio Clínico } \\
\text { Randomizado (II) }\end{array}$ & $\begin{array}{lccc}\text { Analisar os } & \text { efeitos do } \\
\text { relaxamento como intervenção de } \\
\text { enfermagem } & \text { nos } & \text { níveis de } \\
\text { depressão } & \text { de } & \text { mulheres } \\
\text { hospitalizadas } & \text { com } & \text { gravidez de } \\
\text { alto risco. } & & & \end{array}$ & $\begin{array}{l}\text { O relaxamento como intervenção de enfermagem foi } \\
\text { eficaz na redução significativa dos níveis de depressão } \\
\text { de mulheres hospitalizadas com gestações de alto risco. } \\
\text { É uma prática que fortalece o vínculo enfermeiro- } \\
\text { paciente e contribui para uma assistência de qualidade. }\end{array}$ \\
\hline 02 & $\begin{array}{l}\text { Melo et } \\
\text { al. (2016) }\end{array}$ & $\begin{array}{l}\text { Revista de } \\
\text { Enfermagem } \\
\text { UFPE On Line } \\
\quad \text { (B2) }\end{array}$ & $\begin{array}{c}\text { Transversal } \\
\text { qualitativo (VI) }\end{array}$ & $\begin{array}{l}\text { Compreender o cuidado hospitalar } \\
\text { de mulheres que vivenciaram a } \\
\text { gestação de alto risco. }\end{array}$ & $\begin{array}{l}\text { Os enfermeiros obstetras pautaram o cuidado de } \\
\text { enfermagem no respeito à decisão da mulher, no zelo } \\
\text { ao seu corpo e na presença do acompanhante de seu } \\
\text { desejo. A equipe de enfermagem, sensível à escuta e } \\
\text { solícita para com a gestante, valoriza em igual } \\
\text { proporção a realização dos procedimentos técnicos e as } \\
\text { subjetividades que permeiam o ciclo gravídico } \\
\text { puerperal como um todo. }\end{array}$ \\
\hline 03 & $\begin{array}{l}\text { Ferreira } \\
\text { Junior et } \\
\text { al. (2017) }\end{array}$ & $\begin{array}{l}\text { Revista Baiana de } \\
\text { Saúde Pública } \\
\text { (B3) }\end{array}$ & $\begin{array}{c}\text { Transversal } \\
\text { qualitativo (VI) }\end{array}$ & $\begin{array}{l}\text { Conhecer o papel do enfermeiro } \\
\text { no atendimento ao pré-natal de } \\
\text { alto risco realizado na atenção } \\
\text { secundária. }\end{array}$ & $\begin{array}{l}\text { A atuação do enfermeiro precisa está pautada no } \\
\text { acolhimento, na educação em saúde e numa consulta } \\
\text { de enfermagem especializada. }\end{array}$ \\
\hline 04 & $\begin{array}{l}\text { Nazareth } \\
\text { et al. } \\
(2017)\end{array}$ & $\begin{array}{l}\text { Midwifery } \\
\quad \text { (A1) }\end{array}$ & $\begin{array}{c}\text { Transversal } \\
\text { quantitativo (VI) }\end{array}$ & $\begin{array}{l}\text { Comparar dois modelos de } \\
\text { atendimento a gestantes de alto } \\
\text { risco. }\end{array}$ & $\begin{array}{l}\text { O modelo de intervenção a gestantes de alto risco } \\
\text { utilizando tecnologias leves ou não invasivas, obteve } \\
\text { resultados mais favoráveis em comparação com } \\
\text { métodos convencionais. }\end{array}$ \\
\hline 05 & $\begin{array}{l}\text { Errico et } \\
\text { al. }(2018)\end{array}$ & $\begin{array}{l}\text { Revista Brasileira } \\
\text { de Enfermagem } \\
\text { (A2) }\end{array}$ & $\begin{array}{c}\text { Transversal } \\
\text { qualitativo (VI) }\end{array}$ & $\begin{array}{l}\text { Analisar o trabalho do enfermeiro } \\
\text { no pré-natal de alto risco na } \\
\text { atenção secundária. }\end{array}$ & $\begin{array}{l}\text { No pré-natal de alto risco, o enfermeiro pode utilizar a } \\
\text { consulta de enfermagem considerando seu domínio das } \\
\text { tecnologias leves para dialogar com as tecnologias } \\
\text { dura e leve dura. }\end{array}$ \\
\hline 06 & $\begin{array}{l}\text { Nascimen } \\
\text { to et al. } \\
(2018)\end{array}$ & $\begin{array}{l}\text { Revista Prevenção } \\
\text { de Infecção e } \\
\text { Saúde } \\
\text { (B4) }\end{array}$ & $\begin{array}{c}\text { Transversal } \\
\text { qualitativo (VI) }\end{array}$ & $\begin{array}{l}\text { Verificar a assistência de } \\
\text { enfermagem prestada à gestante } \\
\text { de alto risco em maternidade de } \\
\text { município paraibano. }\end{array}$ & $\begin{array}{l}\text { Utilizar a Sistematização da Assistência de } \\
\text { Enfermagem (SAE) possibilita uma melhora efetiva na } \\
\text { qualidade da assistência. Ao acolher gestantes de alto } \\
\text { risco, cabe ao enfermeiro: avaliar os níveis de } \\
\text { complexidade de cuidado estabelecendo as prioridades; } \\
\text { instituir a SAE - histórico, exame físico e gineco- } \\
\text { obstétrico, diagnóstico, prescrição e evolução de } \\
\text { enfermagem - através da avaliação materno-feta. }\end{array}$ \\
\hline
\end{tabular}




\begin{tabular}{|c|c|c|c|c|c|}
\hline 07 & $\begin{array}{l}\text { Soares \& } \\
\text { Higarashi } \\
\text { (2019) }\end{array}$ & $\begin{array}{l}\text { Revista Brasileira } \\
\text { de Enfermagem } \\
\text { (A2) }\end{array}$ & $\begin{array}{c}\text { Transversal } \\
\text { qualitativo (VI) }\end{array}$ & $\begin{array}{l}\text { Discutir os benefícios da } \\
\text { utilização da gestão de caso no } \\
\text { pré-natal de alto risco. }\end{array}$ & $\begin{array}{l}\text { Verificou-se que a gestão de caso identificou } \\
\text { elementos importantes no cuidado às gestantes, os } \\
\text { quais denotaram maior complexidade aos casos; se } \\
\text { mostrou como um espaço pertinente para o enfermeiro } \\
\text { atuar, por se tratar de uma ação interventiva que requer } \\
\text { conhecimento e habilidades específicas. A gestão de } \\
\text { caso proporciona manejo diferenciado em casos } \\
\text { complexos, facilita o fluxo entre os serviços de saúde, } \\
\text { concretizando a integralidade e equidade do cuidado. }\end{array}$ \\
\hline 08 & $\begin{array}{l}\text { Jorge et } \\
\text { al. }(2020)\end{array}$ & $\begin{array}{l}\text { Revista da Rede } \\
\text { de Enfermagem do } \\
\text { Nordeste (B1) }\end{array}$ & $\begin{array}{c}\text { Transversal } \\
\text { qualitativo (VI) }\end{array}$ & $\begin{array}{l}\text { Desvelar as percepções de } \\
\text { enfermeiros sobre assistência } \\
\text { humanizada, no pré-natal de alto } \\
\text { risco. }\end{array}$ & $\begin{array}{l}\text { Constatou-se que a humanização do cuidado consistiu } \\
\text { de ações relacionadas ao acolhimento, atendimento } \\
\text { individualizado, comunicação com gestantes e } \\
\text { estabelecimento de relação de confiança. As principais } \\
\text { práticas de humanização foram as visitas guiadas nas } \\
\text { maternidades; a realização de grupos educacionais; o } \\
\text { uso de métodos não farmacológicos para alívio da dor, } \\
\text { no trabalho de parto; e o incentivo à atuação de } \\
\text { acompanhante. }\end{array}$ \\
\hline 09 & $\begin{array}{l}\text { Teles et } \\
\text { al. }(2020)\end{array}$ & $\begin{array}{l}\text { Revista } \\
\text { Enfermagem em } \\
\text { Foco } \\
\text { (B2) }\end{array}$ & Retrospectivo (VI) & $\begin{array}{l}\text { Identificar os diagnósticos de } \\
\text { enfermagem em um centro de } \\
\text { referência de gestação de alto } \\
\text { risco. }\end{array}$ & $\begin{array}{l}\text { Os principais diagnósticos de enfermagem } \\
\text { classificados foram: conforto prejudicado seguido de } \\
\text { risco de infecção, manutenção ineficaz da saúde e dor } \\
\text { aguda. É importante que os profissionais da } \\
\text { enfermagem tenham uma visão integralizada das } \\
\text { principais necessidades da gestante de alto risco, o que } \\
\text { contribui para a melhora da assistência prestada a esta } \\
\text { mulher. }\end{array}$ \\
\hline 10 & $\begin{array}{l}\text { Souza et } \\
\text { al. }(2020)\end{array}$ & $\begin{array}{l}\text { Revista da Escola } \\
\text { de Enfermagem da } \\
\text { USP } \\
\text { (A1) }\end{array}$ & $\begin{array}{c}\text { Transversal } \\
\text { qualitativo (VI) }\end{array}$ & $\begin{array}{l}\text { Analisar as interações da } \\
\text { enfermagem com a gestante de } \\
\text { alto risco hospitalizada quanto às } \\
\text { possibilidades e limites de uma } \\
\text { assistência norteada pelo princípio } \\
\text { da integralidade. }\end{array}$ & $\begin{array}{l}\text { As práticas de enfermagem são retratadas a partir de } \\
\text { duas categorias temáticas: "Acolhimento: apoio } \\
\text { emocional e informacional" e "Avaliação e } \\
\text { monitoramento do risco gestacional". Os resultados } \\
\text { revelaram intenções de abraçar as mulheres, } \\
\text { fornecendo apoio informal e emocional, mas sob } \\
\text { fragilidades nas relações intersubjetivas. }\end{array}$ \\
\hline 11 & $\begin{array}{l}\text { Ares et } \\
\text { al. }(2021)\end{array}$ & $\begin{array}{l}\text { Revista da Rede } \\
\text { de Enfermagem do } \\
\text { Nordeste (B1) }\end{array}$ & $\begin{array}{c}\text { Transversal } \\
\text { qualitativo (VI) }\end{array}$ & $\begin{array}{l}\text { Conhecer as percepções de } \\
\text { enfermeiras obstétricas sobre os } \\
\text { fatores relacionados com o uso } \\
\text { das tecnologias não invasivas de } \\
\text { cuidado na assistência às } \\
\text { parturientes de alto risco. }\end{array}$ & $\begin{array}{l}\text { Constatou-se que a assistência pré-natal com o enfoque } \\
\text { na autonomia e nos direitos femininos é um fator } \\
\text { facilitador para o uso das tecnologias não invasivas de } \\
\text { cuidado no processo parturitivo das parturientes de } \\
\text { risco. O acesso à informação durante a gestação, } \\
\text { sobretudo de conhecimentos sobre o parto e } \\
\text { nascimento, instrumentaliza as mulheres para o } \\
\text { exercício dos seus direitos na assistência à saúde. }\end{array}$ \\
\hline
\end{tabular}

Nota: *NE - Nível de evidência. Fonte: Dados obtidos no estudo.

\section{Discussão}

Este estudo analisou a produção científica a respeito da assistência de enfermagem prestada à gestante de alto risco, a fim de identificar os principais cuidados prestados pelo enfermeiro a estas gestantes.

Quanto ao desenho metodológico das publicações analisadas, prevaleceu os estudos qualitativos e os transversais quantitativos, que possuem um nível de evidência fraco conforme a classificação dos níveis de evidências de estudos científicos explicitados na metodologia. Porém os métodos analisados são os mais frequentes para este tipo de análise. Pesquisas qualitativas em enfermagem fornecem informações ainda mais refinadas sobre as percepções dos sujeitos analisados, de modo que os dados obtidos lançam luz sobre as barreiras potenciais ao cuidado que não são facilmente reconhecidas em registros administrativos ou clínicos e, assim, fornecem mais detalhes do que algumas pesquisas quantitativas (Doorenbos, 2014).

Neste sentido, esses estudos possibilitaram uma análise minuciosa acerca das diferentes possibilidades de cuidado que o enfermeiro pode exercer durante a assistência às gestantes de alto risco, possibilitando uma análise multidimensional dos fatores elencados e a possibilidade do desenvolvimento de estratégias que melhorem o cuidado desses profissionais frente a 
estas pacientes.

A partir dos artigos analisados nesta revisão, verificou-se que a atuação do enfermeiro na gestação de alto risco está pautada em quatro eixos temáticos. O primeiro eixo identificado se refere à SAE no plano terapêutico das gestantes de risco, reportado por quatro estudos (Errico et al., 2018; Nascimento et al., 2018; Soares \& Higarashi, 2019; Teles et al., 2020). Este instrumento é de fundamental importância uma vez que possibilita uma melhora efetiva da qualidade da assistência entre os enfermeiros, valorizando desta forma a cientificidade da profissão, além de garantir vantagens, como promoção da integração entre a equipe de enfermagem e demais membros da equipe de saúde (Luchtemberg \& Pires, 2016).

No campo da obstetrícia, a SAE tem a finalidade de fornecer acolhimento e apoio a gestante de alto risco, incorporando desta forma, uma assistência efetiva e segura nas diferentes indicações clínicas e obstétricas. No estudo realizado por Nascimento et al. (2018), com sete enfermeiros de uma maternidade de referência da Paraíba, esta ferramenta tinha barreiras para sua implantação, como a falta de recursos humanos, decorrente de uma grande demanda de serviços para os poucos profissionais que trabalham no setor.

O segundo eixo temático identificado nos resultados se refere aos cuidados realizados pelos enfermeiros frente as gestantes de alto risco, sendo retratados como uma ação autônoma baseada no conhecimento científico. Esta categoria foi reportada por cinco estudos presentes na amostra (Araújo et al., 2016; Melo et al., 2016; Errico et al., 2018; Soares \& Higarashi, 2019; Teles et al., 2020). A literatura científica afirma que o processo de enfermagem segue o modelo técnicocientífico que orienta e sistematiza o trabalho do enfermeiro, promovendo uma assistência contínua e qualificada, permitindo a organização das condições para o seu cumprimento e registrando a assistência prestada (Nazareth et al., 2017). O plano de cuidado individualizado realizado no processo de enfermagem mostrou-se eficaz no desenvolvimento de intervenções e no alcance de resultados adequados biologicamente, psicologicamente e socialmente, possibilitando um cuidado diferenciado e humanizado às gestantes de alto risco.

A terceira categoria de atuação do enfermeiro filtrado a partir dos estudos da amostra focou na avaliação e monitoramento do risco gestacional e na execução dos cuidados técnicos-científicos no tratamento das doenças associadas ao risco gestacional durante a gestação, parto e puerpério, a partir da utilização de tecnologias leves. Este eixo foi identificado em cinco publicações presentes na amostra (Nazareth et al., 2017; Errico et al., 2018; Jorge et al., 2020; Souza et al., 2020; Ares et al., 2021).

Outros estudos apontam que estas tecnologias não invasivas de cuidado envolvem, predominantemente, ações que a enfermeiro desenvolve de forma compartilhada com as gestantes e parturientes de alto risco, tais como: oferecimento de apoio emocional e orientações; acompanhamento sensível e contínuo; demonstrações de disponibilidade, acolhimento e empatia; incentivo à participação do acompanhante; valorização das subjetividades; respeito ao protagonismo da mulher; e promoção do bem-estar (Prata et al., 2019).

Em estudo de revisão sistemática, ao se reportar ao tipo de assistência, enfatizou que existe de moderada a alta evidência de que os enfermeiros obstetras utilizam com maiores frequências as tecnologias não invasivas em relação aos profissionais médicos, alcançando semelhantes ou melhores resultados tanto para a gestante e parturiente quanto para o recémnascido (Johantgen et al., 2012).

O quarto eixo temático referente a atuação do enfermeiro às gestantes de alto risco se refere ao processo de educação em saúde. Os artigos que integram este eixo sugerem um modelo de atenção às gestantes de alto risco, visto que o enfermeiro assume a função de ensinar e aconselhar na promoção do comportamento saudável, motivando uma participação ativa, dando suporte à tomada de decisão e fortalecendo a rede social (Melo et al., 2016; Ferreira Junior et al., 2017; Errico et al., 2018).

Considerando a vivência diferenciada da gravidez de alto risco, a utilização de ações educativas ao longo do ciclo gravídico-puerperal é fundamental. As enfermeiras devem assumir a posição de educadoras, que compartilham sua 
compreensão sobre a autonomia e o preparo da mulher, inclusive para seus familiares, para vivenciar a gravidez, o parto e o puerpério (Errico et al., 2018). Os resultados dos estudos analisados demonstraram que essas ações são benéficas e não geram custos extras, possibilitam atendimento individualizado e atendem às necessidades das gestantes.

Pesquisas como a de Rolim et al. (2020), exploram a importância do trabalho do enfermeiro para amenizar o impacto na vida das mulheres e de seus familiares após o diagnóstico de uma gravidez de risco e a influência da ação e da decisão em tempo hábil nos resultados desta gravidez.

A partir de então, o contexto da assistência de enfermagem à gestante de alto risco é repleto de sentimentos de medo e incertezas que exigem conhecimento técnico-científico, compreensão dos problemas enfrentados por essas gestantes e ações oportunas necessárias que contribuam para a consolidação de ações qualificadas e prática assistencial com resultados de saúde satisfatórios tanto para a mãe quanto para o feto.

\section{Conclusão}

Verificou-se que o cuidado à gestante de alto risco ficou evidente por meio de um pré-natal de qualidade, a partir do plano de cuidado individualizado organizado pelo enfermeiro. Além disso, os estudos presentes nesta revisão elencaram a Sistematização da Assistência de Enfermagem como uma ferramenta indispensável no processo de enfermagem, garantindo a gestante uma assistência sistematizada e pautada no conhecimento técnico-científico, afim de que as condições envolvidas que caracterizam a gestação como de alto risco, possam ser acompanhadas e tratadas de forma humanizada e segura.

Desta forma, ressalta-se a importância de realizar outras pesquisas relacionadas à implementação da sistematização de assistência a gestantes de alto risco, para possibilitar uma melhora na qualidade do cuidado e desenvolver as etapas do processo de enfermagem, com a implementação dos cuidados e avaliação dos resultados.

Além disso, verificou-se que o uso de tecnologias leves não invasivas associado ao processo de educação em saúde, pode ser um aliado na busca por um atendimento de qualidade, baseado no acolhimento e escuta qualificada, permeando um vínculo enfermeiro-paciente pautado na ética e no humanismo, fatores estes, importantes e inerentes a enfermagem.

Neste sentido, por meio da colaboração de conhecimentos compartilhados, os enfermeiros obstetras podem melhorar a saúde da mãe e do feto por meio da cogestão de cuidados complexos, uma vez que a colaboração e a comunicação são determinantes importantes na redução da mortalidade em mulheres com gestações de alto risco.

\section{Referências}

Alves, T. O., Nunes, R. L. N., Sena, L. H. A., Alves, F. G., Souza, A. G. S., Salviano, A. M., et al (2021). Gestação de alto risco: epidemiologia e cuidados, uma revisão de literatura. BJRH. 4(4), 14860-14872.

Amorim, T. V., Souza, I. E. O., Moura, M. A. V., Queiroz, A. B. A. \& Salimena, A. M. O. (2017). Perspectivas do cuidado de enfermagem na gestação de alto risco: revisão integrativa. Enferm. glob. 46, 515-528.

Araújo, W. S., Romero, W. G., Zandonade, E., \& Amorim, M. H. C. (2016). Efeitos do relaxamento nos níveis de depressão em mulheres com gestações de alto risco: um ensaio clínico randomizado. Rev. Latino-Am. Enfermagem. 24, e2806.

Ares, L. P. M., Prata, J. A., Progianti, J. M., Pereira, A. L. F., Mouta, R. J. O., Amorim, L. B., et al. (2021). Tecnologias não invasivas na assistência às parturientes de alto risco: percepções de enfermeiras obstétricas. Rev Rene. 22, e61385.

Brasil. Ministério da Saúde (MS). (1998). Lei no 9.610, de 19 de fevereiro de 1998, que altera, atualiza e consolida a legislação sobre direitos autorais e dá outras providências. Diário Oficial da União. Brasília: Ministério da Saúde. http://www.planalto.gov.br/ccivil_03/leis/19610.htm

Brasil. Ministério da Saúde (MS). (2002). Programa de humanização no pré-natal e nascimento: informações para gestores e técnicos. Brasília: Ministério da Saúde. https://bvsms.saude.gov.br/bvs/publicacoes/parto.pdf

Brasil. Ministério da Saúde (MS). (2012). Secretaria de Atenção à Saúde. Departamento de Ações Programáticas Estratégicas. Gestação de alto risco: manual técnico. 5. ed. Brasília: Ministério da Saúde. https://bvsms.saude.gov.br/bvs/publicacoes/manual_tecnico_gestacao_alto_risco.pdf 
Brasil. Ministério da Saúde (MS). Portaria nº 1.020, de 29 de maio de 2013. Institui as diretrizes para a organização da Atenção à Saúde na Gestação de Alto Risco e define os critérios para a implantação e habilitação dos serviços de referência à Atenção à Saúde na Gestação de Alto Risco, incluída a Casa de Gestante, Bebê e Puérpera (CGBP), em conformidade com a 2013. http://bvsms.saude.gov.br/bvs/saudelegis/gm/2013/prt1020_29_05_2013.html

Brasil. (2015). Resolução no 0477 de 14 de abril de 2015. Adaptação sobre a atuação de Enfermeiros na assistência às gestantes, parturientes e puérperas. Diário Oficial da República $\quad$ Federativa do $\quad$ Brasil. $\quad$ Brasília, 375. https://bvsms.saude.gov.br/bvs/publicacoes/manual_tecnico_gestacao_alto_risco.pdf

Doorenbos, A. Z. (2014). Mixed Methods in Nursing Research: An Overview and Practical Examples. Kango Kenkyu, 47(3), $207-217$.

Errico, L. S. P., Bicalho, P. G., Oliveira, T. C. F. L. \& Martins, E. F. (2018). A atuação do enfermeiro no pré-natal de alto risco na perspectiva das necessidades humanas básicas. Rev. Bras. Enferm. 71(3), 1257-1264.

Ferreira Junior, A. R., Filho, J. T. O., Albuquerque, R. A. S., Siqueira, D. D., Rocha, F. A. A. \& Rodrigues, M. E. N. G. (2017). O enfermeiro no pré-natal de alto risco: papel profissional. Rev. baiana saúde pública. 41(3), 650-667.

Giovanni, M. (2013). Rede Cegonha: da concepção à implantação. Curso de Especialização em Gestão Pública da Escola Nacional de Administração Pública ENAP, Brasília.

Higgins, J. \& Green, S. (2011). Cochrane handbook for systematic reviews of interventions version 5.1.0. Chichester: John Wiley \& Sons.

Johantgen, M., Fountain, L., Zangaro, G., Newhouse, R., Stanik-Hutt, J. \& White, K. (2012). Comparison of labor and delivery care provided by certified nurse-midwives and physicans: a systematic review, 1990 to 2008. Women Health. 22(1), 73-81.

Jorge, H. M. F., Silva, R. M. \& Makuch, M. Y. (2020). Atendimento humanizado no pré-natal de alto risco: percepções de enfermeiros. Rev Rene. 21 , e44521.

Luchtemberg, M. N. \& Pires, D. E. P. (2016). Enfermeiros do Serviço de Atendimento Móvel de Urgência: perfil e atividades desenvolvidas. Rev Bras Enferm. 69(2), 213-220.

Melo, M. N., Amorim, T. V., Salimena, A. M. O., Melo, M. C. S. C. \& Souza, I. E. O. (2016). Cuidado hospitalar de mulheres que vivenciaram a gestação de alto risco: contribuições para a enfermagem. Rev enferm UFPE on line. 10(11), 3911-3917.

Moher, D., Shamseer, L., Clarke, M., Ghersi, D., Liberati, A., Petticrew, M., et al (2015). Preferred reporting items for systematic review and meta-analysis protocols (PRISMA-P) 2015 statement. Syst. Rev. 4(1), 1-9.

Nascimento, T. F. H., Araújo, F. N. F., Soares, N. S. C. S., Silva, F. M., Santos, M. F. D. \& Chaves, B. J. P. (2018). Assistência de enfermagem à gestante de alto risco sob a visão do profissional. Rev Pre Infec e Saúde. 4, 68922018.

Nazareth, J. V., Souza, K. V., Beinner, M. A., Barra, J. S., Brüggemannet, O. M. \& Pimenta, A. M. (2017). Special attention to women experiencing high-risk pregnancy: Delivery, care assistance and neonatal outcomes in two Brazilian maternity wards. Midwifery. 53, 42-48.

Pati, D. \& Lorusso, L. N. (2018). How to Write a Systematic Review of the Literature. HERD. 11(1), 15-30.

Prata, J. A., Ares, L. P. M., Vargens, O. M. C., Reis, C. S. C., Pereira, A. L. F. \& Progianti, J. M. (2019). Non-invasive care technologies: nurses' contributions to the demedicalization of health care in a high-risk maternity hospital. Esc Anna Nery. 23(2), e20180259.

Ricci, S. E. (2015). Enfermagem materno-neonatal e saúde da mulher. Guanabara Koogan.

Rios, C. T. F. \& Vieira, N. F. C. (2007). Ações educativas no pré-natal: reflexão sobre a consulta de enfermagem como um espaço para educação em saúde. Ciênc saúde coletiva. $12(2), 477-486$.

Rolim, N. R. F., Gabriel, I. S., Mota, A. S. \& Quental, O. B. (2020). Fatores que contribuem para a classificação da gestação de alto risco: revisão integrativa. Brazilian Journal of Production Engineering. 6(6), 60-68.

Salge, A. K. M., Reis, M. R., Siqueira, K. M., Castral, T. C., Guimarães, J. V. \& Castro, E. C. C. (2017). Associação entre as características macroscópicas do cordão umbilical, gestação de alto risco e repercussões neonatais. Rev esc enferm USP. 51, e032942017.

Soares, L. G. \& Higarashi, I. H. (2019). Gestão de caso como estratégia de cuidado no pré-natal de alto risco. Rev. bras. enferm. 72(3), 692-699.

Soncini, N. C. V., Oliveira, C. M., Viviani, J. C., Gorayeb, R. (2019). Aspectos psicossociais em mulheres brasileiras com gestações de alto e baixo risco. Psicologia, Saúde e Doenças. 20(1), 122-136.

Souza, B. F., Bussadori, J. C. C., Ayres, J. R. C. M., Fabbro, M. R. C. \& Wernet, M. (2020). Enfermagem e gestantes de alto risco hospitalizadas: desafios para integralidade do cuidado. Rev. esc. enferm. USP. 54, e03557.

Souza, M. T., Silva, M. D. \& Carvalho, R. (2010). Integrative review: what is it? How to do it? Einstein (São Paulo). 8(1), $102-106$.

Souza, M. A. O., Souza, N. R., Melo, J. T. S., Xavier, M. A. C. A., Almeida, G. L. \& Santos, I. C. R. V. (2018). Odor evaluation scales for odor in neoplastic wounds: an integrative review. Rev Bras Enferm. 71(5), 2552-2560.

Stillwell, S., Fineout-Overholt, E., Melnyk, B. M. \& Wiliamson, K. M. (2010). Evidence- based practice: step by step. Am J Nurs, $110(5), 41-47$.

Teles, P. A., Costa, E. M., Panobianco, M. S., Gozzo, T. O., Paterra, T. S. V. \& Nunes, L. C. (2019). Diagnósticos de enfermagem mais prevalentes em gestantes de alto risco. Enferm. Foco. 10(3), 119-125.

Wildschut, H. I. J. (2011). Fatores constitucionais e ambientais que levam a uma gravidez de alto risco. In: James DK, Weiner CP, Gonik B, Crowther CA, Robson SC, (Eds.). Gravidez de alto risco: opções de manejo. Amsterdã: Elsevier, 11-28. 